\title{
VANISHING OF HOMOLOGY GROUPS, RICCI ESTIMATE FOR SUBMANIFOLDS AND APPLICATIONS
}

\author{
Antonio Carlos Asperti and Ezio de Araújo Costa
}

\begin{abstract}
In this paper we obtain an estimate for the Ricci curvature and a criterion for the vanishing of the homology groups of compact submanifolds of spheres and Euclidean spaces. This criterion depends on the results of Lawson and Simons [LS], Leung [Le2] and $\mathrm{Xin}[\mathrm{X}]$ on stable currents. As consequences we obtain a topological version of theorems of Cheng and Nakagawa $[\mathrm{CK}]$, Alencar and do Carmo $[\mathrm{AC}]$, and $\mathrm{Xu}[\mathrm{Xu}]$, on hypersurfaces in spheres with constant mean curvature.
\end{abstract}

\section{Introduction}

In [LS], Lawson and Simons obtained a criterion for the vanishing of the homology groups of compact submanifolds of spheres. Latter on and using similar techniques, Leung [Le2] and Xin $[\mathrm{X}]$ were able to extend the results in [LS] to compact submanifolds of Euclidean spaces. Leung also obtained, in [Le1], an estimate for the Ricci curvature of minimal submanifolds of spheres and combined this with the results in [LS] to obtain information on the topology of such submanifolds.

In this paper we follow closely the approach in [Le1]. Firstly we obtain an estimate for the Ricci curvature of submanifolds of a space form which improves Leung's estimates in [Le1] and [Le3]. Next we obtain a criterion, based in the results of $[\mathrm{LS}],[\mathrm{Le} 2]$ and $[\mathrm{X}]$, for the vanishing of the homology groups of compact submanifolds of Euclidean spheres or spaces. Then we combine these results to study the geometry and the topology of such submanifolds.

To state our results, let us fix some notation. We will denote by $f: M^{n} \rightarrow$ $Q_{c}^{n+m}$ an isometric immersion of a connected $n$-dimensional Riemannian manifold $M^{n}$ into a complete, simply connected $(n+m)$-dimensional manifold $Q_{c}^{n+m}$ with constant sectional curvature $c$, where $n \geq 2$ and $m \geq 1$. Let $\vec{H}, H$ and $S$ denote the mean curvature vector of the immersion, its norm and the square of the length

1991 Mathematics Subject Classification: 53C40, 53C42.

Key words: Submanifolds, mean curvature, Ricci curvature, homology groups, conformally flat manifolds.

Received November 22, 1999; revised December 18, 2000. 
of the second fundamental form, respectively. As usual we will denote by $T_{x} M$ the tangent space of $M^{n}$ and by $N_{1}(x)$ the first normal space of the immersion at $x \in M^{n}$. We will make use of the following convention: if $x \in M^{n}$ is such that $\vec{H}(x) \neq 0$, then $\lambda_{1} \leq \lambda_{2} \leq \cdots \leq \lambda_{n}$ will denote the eigenvalues of the Weingarten operator $A_{\xi_{1}}$, in the direction $\xi_{1}=(1 / H) \vec{H}(x)$; if $\vec{H}(x)=0$, just take $\lambda_{i}=0$, $1 \leq i \leq n$, and $\xi_{1}$ any unit vector normal to $M^{n}$ at $x$. Recall that the immersion is quasi-umbilical at $x$ if there exists an orthonormal frame $\xi_{1}, \ldots, \xi_{m}$ in the normal space $T_{x} M^{\perp}$, such that each Weingarten operator $A_{\xi_{\beta}}, \quad 1 \leq \beta \leq m$, has an eigenvalue of multiplicity at least $n-1$; the immersion is quasi-umbilical if it is quasi-umbilical at every $x$ in $M^{n}$.

With this convention, we can state the mentioned estimate for the Ricci curvature of submanifolds and also a sufficient condition for a submanifold $M^{n}$ of $Q_{c}^{n+m}$ to be a conformally flat submanifold with normal curvature tensor $R^{\perp}=0$.

THEOREM 1.1. Let $f: M^{n} \rightarrow Q_{c}^{n+m}$ be an isometric immersion. For every $x \in M^{n}$ and $v \in T_{x} M$, with $\|v\|=1$, we have:

$$
\operatorname{Ric}(v) \geq\left(\frac{n-1}{n}\right)(n c-S)+n H^{2}+(n-2) H\left\langle A_{\xi_{1}} v, v\right\rangle .
$$

(a) If $n \geq 3$ and (1) is an equality for some unit $v \in T_{x} M$, then:

(a1) $f$ is quasi-umbilical at $x, R^{\perp}(x)=0$ and $\operatorname{dim} N_{1}(x) \leq 2$. Moreover, if $\vec{H}(x)=0$ then $\operatorname{dim} N_{1}(x) \leq 1$;

(a2) For any unit vector $\xi \in T_{x} M^{\perp}$ we have $A_{\xi} v=\lambda v$, where $\lambda$ has multiplicity 1 or $n$ as an eigenvalue of $A_{\xi}$.

(b) Let $n \geq 3$. If for each $x$ in $M^{n}$ there exits an unit vector $v$ in $T_{x} M$ such that (1) is an equality, then $M^{n}$ is conformally flat.

Simons [S], Lawson [L] and Chern, do Carmo and Kobayashi [CCK], contributed for the classification of the compact minimal submanifolds $M^{n}$ of the unit sphere $S^{n+1}$, with $S \leq n$. A still open problem is the complete classification of these submanifolds when the ambient space is the unit sphere $S^{n+m}, m \geq 2$. The following corollary is a partial answer to this question.

COROllary 1.2. Let $M^{n}, n \geq 3$, be a compact minimal submanifold of $S^{n+m}$. If $S \leq n$ on $M^{n}$ and the fundamental group of $M^{n}$ is infinite, then $M^{n}$ is a Clifford torus $S_{c_{1}}^{1} \times S_{c_{2}}^{n-1}$ in a totally geodesic submanifold $S^{n+1} \subset S^{n+m}$, where $c_{1}=\sqrt{1 / n}$ and $c_{2}=\sqrt{(n-1) / n}$.

We state now a criterion for the vanishing of the homology groups of compact submanifolds and also other topological results.

THEOREM 1.3. Let $f: M^{n} \rightarrow Q_{c}^{n+m}$ be an isometric immersion, where $M^{n}$ is compact and $c \geq 0$. 
(a) If for some integer $p$ satisfying $2 \leq p \leq n / 2$

$$
S<\frac{n^{2} H^{2}}{n-p}+\frac{n(n-2 p) H \lambda_{1}}{n-p}+n c
$$

holds on $M^{n}$, then the $k$-th homology group $H_{k}(M, \mathbf{Z})=0$, for $p \leq k \leq n-p$.

(b) If

$$
S<\frac{n^{2} H^{2}}{n-1}+\frac{n(n-2) H \lambda_{1}}{n-1}+n c
$$

holds on $M^{n}$, then the fundamental group $\pi_{1}(M)$ of $M^{n}$ is finite and the universal covering $\tilde{M}^{n}$ of $M^{n}$ is compact. Moreover,

(b1) If $n=2$, then $M^{n}$ is diffeomorphic either to the sphere $S^{2}$ or to the real projective space $R P^{2}$, according to $M^{2}$ is orientable or not;

(b2) If $n=3$ and $\pi_{1}(M)=\{0\}$, then $M^{3}$ is diffeomorphic to the sphere $S^{3}$;

(b3) If $n \geq 4$ and $M^{n}$ is orientable when $n$ is even, then $M^{n}$ is homeomorphic to the sphere $S^{n}$.

When $M^{n}$ is complete, we have the following version of $1.3(\mathrm{~b})$. We observe that the main theorem in $[\mathrm{SX}]$ is a consequence of the following result.

COROLlaRY 1.4. Let $M^{n}$ be a complete submanifold of $Q_{c}^{n+m}, c \geq 0$, such that $M^{n}$ is orientable when $n$ is even. If $\sup (S-T)<0$, where $T$ is the right side of (3), then $M^{n}$ is compact and the same conclusions of 1.3(b) are valid.

In [CN], [AC] and [Xu], the authors studied hypersurfaces $M^{n}$ of $S^{n+1}$ with constant mean curvature $H$. In $[\mathrm{CN}]$ it was proved that if $M^{n}$ is complete and sup $S<C(H)$, then $M^{n}$ is totally umbilical; in $[\mathrm{AC}]$ and $[\mathrm{Xu}]$ it was proved that if $M^{n}$ is compact and $S \leq C(H)$, then $M^{n}$ is umbilical or isometric to a torus $S_{c_{1}}^{1} \times S_{c_{2}}^{n-1}$. In our next theorem, we remove the condition $H=$ constant and obtain a topological-geometrical version of this statement, in any codimension.

THEOREM 1.5. Let $f: M^{n} \rightarrow S^{n+m}$ be an isometric immersion, where $M^{n}$ is compact. Let $S \leq C(H)$ on $M^{n}$, where

$$
C(H)=n+\frac{n^{3} H^{2}}{2(n-1)}-\frac{n(n-2) H}{2(n-1)} \sqrt{n^{2} H^{2}+4(n-1)} .
$$

Then the Ricci curvature of $M^{n}$ is nonnegative and we have only two possibilities, (a) and (b):

(a) $\pi_{1}(M)$ is finite. In this case we have:

(a1) If $n=2$, then $M^{2}$ is diffeomorphic to $S^{2}$ or to $R P^{2}$;

(a2) If $n=3$ and $\pi_{1}(M)=\{0\}$, then $M^{3}$ is diffeomorphic to $S^{3}$;

(a3) Let $n \geq 4$ and assume that $H \neq 0$ when $S=C(H)$. If $M^{n}$ is orientable when $n$ is even, then $M^{n}$ is homeomorphic to $S^{n}$. 
(b) $\pi_{1}(M)$ is infinite. In this case, $S=C(H)$ on $M^{n}$ and:

(b1) If $n=2$, then $M^{2}$ is flat;

(b2) If $n \geq 3$, and $M^{n}$ is orientable, then the codimension $m$ of $f$ can be reduced to 1 , the mean curvature $H$ is constant and $f$ has two constant principal curvatures, $\mu_{1}$ and $\mu_{2}$, with multiplicities 1 and $n-1$, respectivelly, such that $\mu_{1} \cdot \mu_{2}=-1$. Consequentely, $M^{n}$ is isometric to a torus $S_{c_{1}}^{1} \times S_{c_{2}}^{n-1}$ in $S^{n+1}$, where $c_{i}=1+\mu_{i}^{2}, i=1,2$.

Note that the pinching constant $C(H)$ for $S$ in Theorem 1.5 depends on $H$ (and $n$ ) and then depends of a specific immersion. A pinching constant depending only on $n$ was firstly obtained by Lawson and Simons in [LS]. There it is proved that if $M^{n}$ is a compact submanifold of $S^{n+m}$ such that $n \geq 5$ and $S<2 \sqrt{n-1}$ on $M^{n}$, then $M^{n}$ is homeomorphic to $S^{n}$. Related to this, recently Hou [Ho] proved the following rigidity result: let $M^{n}$ be a compact submanifold of $S^{n+m}$ with non-zero parallel mean curvature vector. If $n \geq 8$ or $m \leq 2$ and $S \leq 2 \sqrt{n-1}$ on $M^{n}$, then the codimension can be reduced to 1 and $M^{n}$ is either umbilical or isometric to $S_{c_{1}}^{1} \times S_{c_{2}}^{n-1}$, where $c_{1}=1+\sqrt{n-1}$ and $c_{2}=1+1 / \sqrt{n-1}$. The next corollary is an extension of the theorem in [LS] and a topological-geometrical version of the result in $[\mathrm{Ho}]$.

COROLlaRY 1.6. Let $M^{n}$ be a compact submanifold of $S^{n+m}$ such that $S \leq 2 \sqrt{n-1}$ on $M^{n}$. Then the Ricci curvature of $M^{n}$ is nonnegative and we have only two possibilities (a) and (b):

(a) There exists a point $x$ in $M^{n}$ such that $\operatorname{Ric}(v)>0$, for all unit vector $v$ in $T_{x} M$. In this case $M^{n}$ admits a metric of positive Ricci curvature and

(a1) If $n=2$, then $M^{n}$ is diffeomorphic to $S^{2}$ or to $R P^{2}$;

(a2) If $n=3$, then $M^{3}$ is orientable with $H_{2}(M, \mathbf{Z})=\{0\}$ and, if $\pi(M)=\{0\}$, then $M^{3}$ is diffeomorphic to $S^{3}$; to $S^{n}$.

(a3) If $n \geq 4$ and $M^{n}$ is orientable when $n$ is even, then $M^{n}$ is homeomorphic

(b) For each point $x$ in $M^{n}$, there exists an unit vector $v$ in $T_{x} M$ such that $\operatorname{Ric}(v)=0$. In this case $S=2 \sqrt{n-1}$ and $n^{2} H^{2}=n \sqrt{n-1}-2(n-1)$ on $M^{n}$ and

(b1) If $n=2$, then $M^{2}$ is flat and minimal. In particular, if $m=1, M^{2}$ is isometric to a torus $S_{2}^{1} \times S_{2}^{1}$;

(b2) If $n \geq 3$, then the codimension $m$ can be reduced to 1 and, if $M^{n}$ is orientable, $M^{n}$ is isometric to the torus $S_{c_{1}}^{1} \times S_{c_{2}}^{n-1}$, where $c_{1}=1+\sqrt{n-1}, c_{2}=$ $1+1 / \sqrt{n-1}$.

We observe that the result in (a1) was obtained by Wei [W].

\section{Notations and preliminary lemmas}

Let $M=M^{n}, n \geq 2$, be a connected $n$-dimensional Riemannian manifold. We denote by $\langle$,$\rangle and by \|\|$ the metric and norm, respectively. Let $R$ denote the curvature tensor of $M$. Then the Ricci tensor is defined by 


$$
\operatorname{Ric}(v, w)=\sum_{i=1}^{n}\left\langle R\left(v_{i}, v\right) w, v_{i}\right\rangle
$$

where $v, w$ are in the tangent space $T_{x} M$ of $M$ at $x$, and $\left\{v_{i}\right\}_{i=1}^{n}$ is any orthonormal basis of $T_{x} M$. The Ricci curvature $\operatorname{Ric}(v)$ in the unit direction $v \in T_{x} M$ and the scalar curvature $\tau$ of $M$ at $x$ are given, respectively, by

$$
\operatorname{Ric}(v)=\langle Q v, v\rangle, \quad \tau=\operatorname{tr} Q,
$$

where $Q: T_{x} M \rightarrow T_{x} M$ is defined by

$$
\langle Q v, w\rangle=\operatorname{Ric}(v, w) .
$$

Let $f: M^{n} \rightarrow Q_{c}^{n+m}, m \geq 1$, be an isometric immersion, where $Q_{c}^{n+m}$ is a complete, simply connected $(n+m)$-dimensional manifold with constant sectional curvature $c$. For each $x \in M^{n}$, let $\left(T_{x} M\right)^{\perp}$ denote the normal space of $f$ at $x$, and $\alpha: T_{x} M \times T_{x} M \rightarrow\left(T_{x} M\right)^{\perp}$ denote the second fundamental form of $f$ at $x$.

If $\left\{\xi_{\beta}\right\}_{\beta=1}^{m}$ is any orthonormal basis of $\left(T_{x} M\right)^{\perp}$, then the Weingarten operator $A_{\xi_{\beta}}$, in the normal direction $\xi_{\beta}$, is defined by

$$
\left\langle A_{\xi_{\beta}} v, w\right\rangle=\left\langle\alpha(v, w), \xi_{\beta}\right\rangle, \quad v, w \in T_{x} M .
$$

The mean curvature vector $\vec{H}=\vec{H}(x)$ at $x$ and its norm are defined by

$$
\vec{H}=\frac{1}{n} \sum_{\beta=1}^{m}\left(\operatorname{tr} A_{\xi_{\beta}}\right) \xi_{\beta}, \quad H=\|\vec{H}\| .
$$

The square of the length of the second fundamental form of $f$ at $x$ is defined by

$$
S=\sum_{\beta=1}^{m} \operatorname{tr} A_{\xi_{\beta}}^{2} .
$$

We then have the following relations:

$$
\sum_{\beta=1}^{m} A_{\xi_{\beta}}^{2}-\sum_{\beta=1}^{m}\left(\operatorname{tr} A_{\xi_{\beta}}\right) A_{\xi_{\beta}}=-Q+(n-1) c I,
$$

where $I: T_{x} M \rightarrow T_{x} M$ is the identity map, and

$$
S=-\tau+n^{2} H^{2}+n(n-1) c .
$$

We now present three lemmas which will be used in the next sections.

Lemma 2.1. Let $V$ be a real vector space of dimension $n \geq 2$ with an inner product $\langle$,$\rangle and norm \|\|$. Let $A: V \rightarrow V$ be a symmetric linear map and let $H$ be such that $\operatorname{tr} A=n H$. If $\lambda_{1} \leq \lambda_{2} \leq \cdots \leq \lambda_{n}$ are the eigenvalues of $A$, then for any unit vector $v \in V$ we have

(a) $\left\langle A^{2} v, v\right\rangle \leq((n-1) / n)\left[\operatorname{tr} A^{2}-n H^{2}\right]+2 H\langle A v, v\rangle-H^{2}$. If $n \geq 3$ and the equality occurs in (a), for some unit vector $v$, then $A v=\lambda_{j} v$ where $j$ satisfies 
$\left|\lambda_{j}-H\right|=\max \left\{\left|\lambda_{i}-H\right|, i=1, \ldots, n\right\}$. Also $\lambda_{k}=\lambda_{l}$ for all $k, l \neq j$, and $A w=\lambda_{k} w$, $k \neq j$, for any $w$ orthogonal to $v$.

(b) $\langle A v, v\rangle \geq \lambda_{1} \geq H-\sqrt{((n-1) / n)\left[\operatorname{tr} A^{2}-n H^{2}\right]}$. Moreover, if $\lambda_{1}=H-$ $\sqrt{((n-1) / n)\left[\operatorname{tr} A^{2}-n H^{2}\right]}$, then $\lambda_{i}=\lambda_{2}$ for $i \geq 2$.

Proof. Let $\left\{v_{i}\right\}_{i=1}^{n}$ be an orthonormal basis of $V$ such that they are eigenvectors of $A$, where $A v_{i}=\lambda_{i} v_{i}$, for all $i$. Assume first that $\operatorname{tr} A=n H=0$ and let $\lambda_{j}$ be such that $\lambda_{j}^{2}=\max \left\{\lambda_{i}^{2}, i=1, \ldots, n\right\}$. Since $\lambda_{j}=-\sum_{i \neq j} \lambda_{i}$, then

$$
\lambda_{j}^{2}=\left(\sum_{i \neq j} \lambda_{i}\right)^{2} \leq(n-1) \sum_{i \neq j} \lambda_{i}^{2}
$$

and

$$
\lambda_{j}^{2} \leq\left(\frac{n-1}{n}\right) \operatorname{tr} A^{2} .
$$

If $v$ is a unit vector, it is clear that

$$
\left\langle A^{2} v, v\right\rangle \leq \lambda_{j}^{2} \leq\left(\frac{n-1}{n}\right) \operatorname{tr} A^{2},
$$

and then the first part of (a) follows for $H=0$. Now assume that $n \geq 3$ and let $v=\sum_{i=1}^{n} a_{i} v_{i}$ be a unit vector such that $\left\langle A^{2} v, v\right\rangle=((n-1) / n) \operatorname{tr} A^{2}$. Then $\left\langle A^{2} v, v\right\rangle=\lambda_{j}^{2}=((n-1) / n) \operatorname{tr} A^{2}$ and (11) is an equality. Since

$$
\left(\sum_{i \neq j} \lambda_{j}\right)^{2}=(n-1) \sum_{i \neq j} \lambda_{i}^{2}-\sum_{\substack{k, l \neq j \\ k<l}}\left(\lambda_{k}-\lambda_{l}\right)^{2}
$$

we have by (11) that $\lambda_{k}=\lambda_{l}$ for all $k, l \neq j$. Also for any $k \neq j$ we have

$$
\lambda_{j}^{2}=\left\langle A^{2} v, v\right\rangle=\sum_{i=1}^{n} a_{i}^{2} \lambda_{i}^{2}=\lambda_{k}^{2}\left(1-a_{j}\right)^{2}+a_{j}^{2} \lambda_{j}^{2},
$$

that is, $\lambda_{j}^{2}\left(1-a_{j}^{2}\right)=\lambda_{k}^{2}\left(1-a_{j}^{2}\right)$. If $a_{j}^{2}=1$, then $a_{i}=0$ for $i \neq j$ and $v= \pm v_{j}$. If $a_{j}^{2} \neq 1$, it follows that $\lambda_{i}^{2}=\lambda_{j}^{2}$ for all $i=1, \ldots, n$ and by the equality in (11), this implies that $\lambda_{j}^{2}=(n-1) \lambda_{j}^{2}$. Since $n \geq 3, \lambda_{i}=0$ for all $i=1, \ldots, n$. In any case, we have $A v=\lambda_{j} v$. Now let $w$ be orthogonal to $v$. If $v= \pm v_{j}$, then $w=\sum_{i \neq j} b_{i} v_{i}$ and so $A w=\lambda_{k} w, \forall k \neq j$; otherwise every $\lambda_{i}=0$ and then $A w=0$. For the part (b), note that $\langle A v, v\rangle \geq \lambda_{1}$. Since $\lambda_{1} \leq H=0$ and $\lambda_{1}^{2} \leq$ $((n-1) / n) \operatorname{tr} A^{2}$, then

$$
\lambda_{1} \geq-\sqrt{\left(\frac{n-1}{n}\right) \operatorname{tr} A^{2}},
$$

which is the desired result (b) for $H=0$. 
Suppose now that $H \neq 0$ and let $B=A-H I$, where $I: V \rightarrow V$ is the identity map. Then $\operatorname{tr} B=0, B^{2}=A^{2}-2 H A+H^{2} I$ and $\operatorname{tr} B^{2}=\operatorname{tr} A^{2}-n H^{2}$. The result (a) follows immediately by applying the case $H=0$ to $B$. Since $\langle A v, v\rangle \geq \lambda_{1}$ and $H \geq \lambda_{1}$, choose $v=v_{1}$ in (a) for $B=A-H I$. Then

$$
\left(\lambda_{1}-H\right)^{2} \leq\left(\frac{n-1}{n}\right)\left[\operatorname{tr} A^{2}-n H^{2}\right]
$$

and the first part of (b) follows for $H \neq 0$. For the proof of the second part of (b), since $(n-1) \sum\left(b_{i}\right)^{2}=\left(\sum b_{i}\right)^{2}$ holds, where $b_{i}=\lambda_{i}-H$ and $i \geq 2$, then we have $\lambda_{i}-H=\lambda_{2}-H$ for all $i \geq 2$. This completes the proof.

Lemma 2.2. Let $M^{n}, n \geq 4$, be a connected, compact $n$-dimensional Riemannian manifold such that $M^{n}$ is orientable if $n$ is even, and let $\tilde{M}^{n}$ be the universal covering of $M^{n}$. If $\pi_{1}(M)$ is finite and $H_{p}(M, \mathbf{Z}) \simeq H_{p}(\tilde{M}, \mathbf{Z})=\{0\}$ for all $p=2, \ldots, n-2$, then $M^{n}$ is homeomorphic to a sphere $S^{n}$.

Proof. Firstly we observe that $M^{n}$ is orientable if $n$ is odd. In fact, if not, then $H_{n}(M, \mathbf{Z})=\{0\}$, see Corollary 7.12 of $[\mathrm{B}]$. But the Euler characteristic $\chi(M)$ of $M^{n}$ is zero and also $\chi(M)=b_{0}-b_{1}+\cdots+b_{n-1}-b_{n}$, where $b_{i}=$ rank $H_{i}(M, \mathbf{Z})$. Since $\pi_{1}(M)$ is finite, $b_{1}=0$ and then $\chi(M)=1+b_{n-1} \geq 1$, a contradiction. Now the torsion part of $H_{1}(M, \mathbf{Z})$ is $H_{1}(M, \mathbf{Z})$, because it is finite. But by the universal coefficient theorem, see [B, p. 282], the cohomology group $H^{i}(M, \mathbf{Z})$ is isomorphic to $F_{i} \oplus T_{i-1}$, where $F_{i}$ and $T_{i}$ are the free and torsion parts of $H_{i}(M, \mathbf{Z})$. By Poincaré duality, $H_{1}(M, \mathbf{Z})$ is isomorphic to $H^{n-1}(M, \mathbf{Z})$ and so $H_{1}(M, \mathbf{Z})=\{0\}$. Again by the universal coefficient theorem, $H^{1}(M, \mathbf{Z})=\{0\}$ and, by Poincaré duality, $H_{n-1}(M, \mathbf{Z})=\{0\}$. Then $M^{n}$ is a homology sphere and the same arguments applied to $\tilde{M}^{n}$ tell us that $\tilde{M}^{n}$ is a homology sphere. Since $\pi_{1}(\tilde{M})=\{0\}$, by standards arguments using the Hurewicz isomorphism theorem and Whitehead theorem, see [Sp, p. 398], we conclude that $\tilde{M}^{n}$ is indeed a homotopy sphere. By the generalized Poincare conjecture for $n \geq 4, \tilde{M}^{n}$ is homeomorphic to a sphere. Then we have a homology sphere $M^{n}$ which is covered by a sphere $\tilde{M}^{n}$ and so, by a theorem of Sjerve [Sj], $\pi_{1}(M)=\{0\}$ and hence $M^{n}$ is also homeomorphic to a sphere. This concludes the proof.

Lemma 2.3. Let $V$ be a real vector space of dimension $n \geq 2$ with inner product $\langle$,$\rangle and let A: V \rightarrow V$ be a symmetric linear map with $\operatorname{tr} A=n H$. Let $p$ be a positive integer such that $p \leq n / 2$ and let $\left\{v_{1}, \ldots, v_{p}, v_{p+1}, \ldots, v_{n}\right\}$ be an orthonormal basis of $V$. Denote by

$$
\Theta=\left\{\begin{array}{l}
\operatorname{tr} A^{2}-(n+1) H^{2}-(n-2) H\left\langle A v_{1}, v_{1}\right\rangle, \quad \text { if } p=1, \\
\frac{p(n-p)}{n} \operatorname{tr} A^{2}-p n H^{2}-(n-2 p) H \sum_{i=1}^{p}\left\langle A v_{1}, v_{1}\right\rangle, \quad \text { if } p>1 .
\end{array}\right.
$$


Then

$$
\left(\sum_{i}\left\langle A v_{i}, v_{i}\right\rangle\right)^{2}-n H \sum_{i}\left\langle A v_{i}, v_{i}\right\rangle+2 \sum_{i, k}\left\langle A v_{i}, v_{k}\right\rangle^{2} \leq \Theta,
$$

where, $i=1, \ldots, p$ and $k=p+1, \ldots, n$.

Proof. Since $A$ is symmetric, clearly

$$
\operatorname{tr} A^{2} \geq \sum_{i}\left\langle A v_{i}, v_{i}\right\rangle^{2}+\sum_{k}\left\langle A v_{k}, v_{k}\right\rangle^{2}+2 \sum_{i, k}\left\langle A v_{i}, v_{k}\right\rangle^{2} .
$$

Assume first that $\operatorname{tr} A=n H=0$. If $p=1$, we have

$$
\operatorname{tr} A^{2} \geq\left\langle A v_{1}, v_{1}\right\rangle^{2}+2 \sum_{k}\left\langle A v_{1}, v_{k}\right\rangle^{2}
$$

and the lemma follows for $H=0$ and $p=1$. If $2 \leq p \leq n / 2$, then $p(n-p) \geq n$. On the other hand, we have that

$$
\operatorname{tr} A^{2} \geq \frac{1}{p}\left[\sum_{i}\left\langle A v_{i}, v_{i}\right\rangle\right]^{2}+\frac{1}{n-p}\left[\sum_{k}\left\langle A v_{k}, v_{k}\right\rangle\right]^{2}+2 \sum_{i, k}\left\langle A v_{i}, v_{k}\right\rangle^{2} .
$$

Since $\operatorname{tr} A=\sum_{i}\left\langle A v_{i}, v_{i}\right\rangle+\sum_{k}\left\langle A v_{k}, v_{k}\right\rangle=0$, then from the above inequalities it follows that

$$
\frac{p(n-p)}{n} \operatorname{tr} A^{2} \geq\left[\sum_{i}\left\langle A v_{i}, v_{i}\right\rangle\right]^{2}+2 \sum_{i, k}\left\langle A v_{i}, v_{k}\right\rangle^{2},
$$

which is the desired result (13) for $H=0$ and $p>1$. For $H \neq 0$, let $B=$ $A-H I$, where $I: V \rightarrow V$ is the identity map. Then $\operatorname{tr} B=0, B^{2}=A^{2}-$ $2 H A+A^{2} I$ and $\operatorname{tr} B^{2}=\operatorname{tr} A^{2}-n H^{2}$. The result (13) follows immediately by applying the case $H=0$ to $B$.

\section{Proofs of Theorem 1.1 and Corollary 1.2}

The following theorem, which was proved by Lawson and Simons [LS] in the case $c>0$ and, independently, by Leung [Le2], Wei $[\mathrm{W}]$ and Xin $[\mathrm{X}]$ in the case $c=0$, is essential in the proof of Theorem 1.1.

THEOREM 3.1. Let $M^{n}$ be a compact manifold isometrically immersed in $Q_{c}^{n+m}, c \geq 0$. Denote by $\alpha$ the second fundamental form of the immersion and let $p$ and $q$ be positive integers such that $p+q=n$. Suppose that at each point $x$ of $M^{n}$ and for all orthonormal basis $\left\{v_{1}, \ldots, v_{p}, v_{p+1}, \ldots, v_{n}\right\}$ of $T_{x} M$, the following condition is valid 


$$
\Phi:=\sum_{i=1}^{p} \sum_{k=p+1}^{n}\left[2\left\|\alpha\left(v_{i}, v_{k}\right)\right\|^{2}-\left\langle\alpha\left(v_{i}, v_{i}\right), \alpha\left(v_{k}, v_{k}\right)\right\rangle\right]<p q c .
$$

Then $H_{p}(M, \mathbf{Z})=H_{q}(M, \mathbf{Z})=\{0\}$ and $\pi_{1}(M)=\{0\}$ if $p=1$.

Proof of Theorem 1.1. Let $x \in M^{n}$. If $\vec{H}(x) \neq 0$, let $\xi_{1}, \ldots, \xi_{m}$ be an orthonormal basis of $T_{x} M^{\perp}$ such that $\xi_{1}=(1 / H) \vec{H}(x)$. Since $\operatorname{tr} A_{\xi_{1}}=n H$ and $\operatorname{tr} A_{\xi_{\beta}}=0$ for $\beta \geq 2$, it follows from (9) that

$$
\begin{gathered}
\sum_{\beta=2}^{m}\left\langle A_{\xi_{\beta}}^{2} v, v\right\rangle+\left\langle A_{\xi_{1}} v, v\right\rangle-n H\left\langle A_{\xi_{1}} v, v\right\rangle \\
=-\operatorname{Ric}(v)+(n-1) c .
\end{gathered}
$$

If $\vec{H}(x)=0$, let $\left\{\xi_{\beta}\right\}_{\beta=1}^{m}$ be any orthonormal basis of $\left(T_{x} M\right)^{\perp}$ and then (16) also follows from (9). Now applying Lemma 2.1 to each $A_{\xi_{\beta}}$ in (16), we get (1).

(a) Suppose that $n \geq 3$ and that (1) is an equality for some unit vector $v$ in $T_{x} M$. By (8) and (16) we have

$$
\begin{aligned}
\sum_{\beta=2}^{m}[ & \left.\left\langle A_{\xi_{\beta}}^{2} v, v\right\rangle-\left(\frac{n-1}{n}\right) \operatorname{tr} A_{\xi_{\beta}}^{2}\right] \\
& +\left[\left\langle A_{\xi_{1}}^{2} v, v\right\rangle-\left(\frac{n-1}{n}\right) \operatorname{tr} A_{\xi_{1}}^{2}-2 H\left\langle A_{\xi_{1}} v, v\right\rangle+n H^{2}\right]=0 .
\end{aligned}
$$

Also by Lemma 2.1(a), we see that in (17), the expressions between the brackets are null. Therefore, again by 2.1(a), $v$ is an eigenvector for all $A_{\xi_{\beta}}$ and, for any $w$ orthogonal to $v$ in $T_{x} M$, we have $A_{\xi_{\beta}} w=\lambda_{\beta} w$. That is, $f$ is quasi-umbilical at $x$ and also $R_{x}^{\perp}=0$. Let $v_{1}=v, v_{2}, \ldots, v_{n}$ be an orthonormal basis of $T_{x} M$ such that $A_{\xi_{\beta}} v_{1}=\mu_{\beta} v_{1}$ and $A_{\xi_{\beta}} v_{i}=\lambda_{\beta} v_{i}$ for all $i \geq 2$. The first normal space $N_{1}(x)$ is spanned by $\alpha\left(v_{i}, v_{i}\right), i=1, \ldots, n$. For $i \geq 2$ we have that $\alpha\left(v_{i}, v_{i}\right)=$ $\sum_{\beta=1}^{m} \lambda_{\beta} \xi_{\beta}=\xi$ and, for $i=1, \alpha\left(v_{1}, v_{1}\right)=\sum_{\beta=1}^{m} \mu_{\beta} \xi_{\beta}=\xi_{0}$. This shows that the dimension of $N_{1}(x)$ is at most two. In particular, if $\vec{H}(x)=0$ then $(n-1) \xi+$ $\xi_{0}=0$ and the dimension of $N_{1}(x)$ is at most one. This proves (a1). For (a2), let $\xi=\sum_{\beta} a_{\beta} \xi_{\beta}$ be a unit vector in $T_{x} M^{\perp}$. Then $A_{\xi} v=\lambda v$, where $\lambda=\sum_{\beta} a_{\beta} \mu_{\beta}$ and the multiplicity of $\lambda$ is 1 or $n$.

(b) Let $n \geq 3$. If $n \geq 4$, then (b) follows from (a) and from a result of [CY] on conformally flat submanifolds. If $n=3$, by a well known characterization of conformally flat manifolds, see [D, p. 108], we have to show that the tensor $\gamma(X)=Q(X)-\tau X / 4$ satisfies the Codazzi's equation $\left(\nabla_{X} \gamma\right)(Y)=\left(\nabla_{Y} \gamma\right)(X)$, $X, Y \in T M$. Given $x$ in $M^{3}$, since $R^{\perp}=0$ there exists a connected open set $V$ around $x$ in $M^{3}$, where we can take a normal orthonormal frame field $\xi_{1}, \ldots, \xi_{m}$ such that $\nabla^{\perp} \xi_{\beta}=0, \beta \geq 1$. By part (a), there exists an orthonormal basis $\left\{X_{1}, X_{2}, X_{3}\right\}$ of $T_{x} M$ such that $A_{\xi_{\beta}} X_{1}=\mu_{\beta} X_{1}$ and $A_{\xi_{\beta}} X_{i}=\lambda_{\beta} X_{i}, \beta \geq 1, i=2,3$. Observe that $\operatorname{tr} A_{\xi_{\beta}}=\mu_{\beta}+2 \lambda_{\beta}$ and $\tau=\operatorname{Ric}\left(X_{1}\right)+2 \operatorname{Ric}\left(X_{i}\right), i=2,3$. Then by (9) we obtain that $\operatorname{Ric}\left(X_{1}\right)=2 c+2 \sum_{\beta} \lambda_{\beta} \mu_{\beta}$ and 


$$
\operatorname{Ric}\left(X_{i}\right)=2 c+\sum_{\beta}\left(\lambda_{\beta} \mu_{\beta}+\lambda_{\beta}^{2}\right), \quad i=2,3, \quad \beta \geq 1 .
$$

Taking these to the definition of $\gamma$, gives

$$
\gamma\left(X_{1}\right)=\left(\frac{c}{2}+\sum_{\beta} \lambda_{\beta}\left(\mu_{\beta}-\frac{\lambda_{\beta}}{2}\right)\right) X_{1}, \quad \gamma\left(X_{i}\right)=\left(\frac{c}{2}+\frac{1}{2} \sum_{\beta} \lambda_{\beta}^{2}\right) X_{i}, \quad i=2,3 .
$$

Therefore $\gamma=(c / 2) I+\sum_{\beta} \lambda_{\beta}\left(A_{\xi_{\beta}}-\left(\lambda_{\beta} / 2\right) I\right)$.

Assume now that $m=1$ (the case $m \geq 2$ is similar) and consider $A=$ $\left\{y \in V: \lambda_{1}=\mu_{1}\right\}$ and $B=\left\{y \in V: \lambda_{1} \neq \mu_{1}\right\}$ (for the general $m$, we have to consider $2^{m}$ of such subsets). Clearly $\operatorname{int}(A) \cup B$ is open and dense in $V$ and, in particular, $\lambda_{1}$ and $\mu_{1}$ are smooth in this set. Now using the Codazzi's equations for each $H_{\xi_{\beta}}$ in $\operatorname{int}(A) \cup B$, it follows that $\gamma$ satisfies the Codazzi's equations in $\operatorname{int}(A) \cup B$ and therefore in $V$. This proves that $M^{3}$ is conformally flat.

Proof of Corollary 1.2. Let $M^{n}$ be a compact and minimal submanifold of $S^{n+m}$ such that $S \leq n$ on $M^{n}$ and with infinite $\pi_{1}(M)$. By (1) we have that $M^{n}$ has everywhere nonnegative Ricci curvature and we claim that for all $x$ in $M^{n}$, there exists an unit $v$ in $T_{x} M$ such that $\operatorname{Ric}(v)=0$. In fact, if there exists $x$ in $M^{n}$ such that $\operatorname{Ric}(v)>0$ for all unit vector $v$ in $T_{x} M$, then by Aubin's theorem [A, p. 397], $M^{n}$ has a metric with positive Ricci curvature and by Bonnet-Myers' theorem, $\pi_{1}(M)$ is finite, a contradiction which proves our claim. Then by (1) and 1.1(a1), $S=n, R^{\perp}=0$ and the dimension of $N_{1}$ is 1 on $M^{n}$. The corollary now follows immediately from Theorem 3 of $[\mathrm{K}]$.

\section{Proofs of Theorem 1.3 and Corollary 1.4}

Proof of Theorem 1.3. (a) Let $2 \leq p \leq n / 2, x \in M^{n}$ and let $\left\{v_{1}, \ldots, v_{n}\right\}$ be an orthonormal basis of $T_{x} M$. Let $\left\{\xi_{\beta}\right\}_{\beta=1}^{m}$ be an orthonormal basis of $T_{x} M^{\perp}$ such that $\xi_{1}=(1 / H) \vec{H}(x)$ if $\vec{H}(x) \neq 0$. Since $\operatorname{tr} A_{\xi_{1}}=n H$ and $\operatorname{tr} A_{\xi_{\beta}}=0, \beta \geq 2$, we have

$$
\begin{aligned}
\Phi= & \sum_{i, k}\left[2\left\|\alpha\left(v_{i}, v_{k}\right)\right\|^{2}-\left\langle\alpha\left(v_{i}, v_{i}\right), \alpha\left(v_{k}, v_{k}\right)\right\rangle\right] \\
= & \sum_{i, k} \sum_{\beta \geq 1}\left[2\left\langle A_{\xi_{\beta}} v_{i}, v_{k}\right\rangle^{2}-\left\langle A_{\xi_{\beta}} v_{i}, v_{i}\right\rangle\left\langle A_{\xi_{\beta}} v_{k}, v_{k}\right\rangle\right] \\
= & 2 \sum_{i, k} \sum_{\beta>1}\left\langle A_{\xi_{\beta}} v_{i}, v_{k}\right\rangle^{2}+\sum_{\beta>1}\left[\sum_{i}\left\langle A_{\xi_{\beta}} v_{i}, v_{i}\right\rangle\right]^{2} \\
& +2 \sum_{i, k}\left\langle A_{\xi_{1}} v_{i}, v_{k}\right\rangle^{2}+\left[\sum_{i}\left\langle A_{\xi_{1}} v_{i}, v_{i}\right\rangle\right]^{2}-n H \sum_{i}\left\langle A_{\xi_{1}} v_{i}, v_{i}\right\rangle,
\end{aligned}
$$


for $i=1, \ldots, p$ and $k=p+1, \ldots, n$. Applying Lemma 2.3 to each $A_{\xi_{\beta}}$, we get

$$
\Phi \leq \frac{p(n-p)}{n} S-p n H^{2}-(n-2 p) H \sum_{i}\left\langle A_{\xi_{1}} v_{i}, v_{i}\right\rangle .
$$

Applying Lemma 2.1(b) to (19), we get

$$
\Phi \leq \frac{p(n-p)}{n} S-p n H^{2}-p(n-2 p) H \lambda_{1},
$$

and then the condition (15) of Theorem 3.1 is valid if

$$
S<\frac{n^{2} H^{2}}{n-p}+\frac{n(n-2 p)}{n-p} H \lambda_{1}+n c
$$

on $M^{n}$. On the other hand, for $p \leq k \leq n-p$, we have that

$$
\frac{n^{2} H^{2}}{n-p}+\frac{n(n-2 p)}{n-p} H \lambda_{1}+n c \leq \frac{n^{2} H^{2}}{n-k}+\frac{n(n-2 k)}{n-k} H \lambda_{1}+n c
$$

and it follows from Theorem 3.1 and $(20)$ that $H_{k}(M, \mathbf{Z})=\{0\}$, which proves $1.3(\mathrm{a})$.

(b) Let $x \in M^{n}$ and $v$ be a unit vector in $T_{x} M$. Since $\left\langle A_{\xi_{1}} v, v\right\rangle \geq \lambda_{1}$ when $H \neq 0$, by (1) we have that

$$
\operatorname{Ric}(v) \geq\left(\frac{n-1}{n}\right)(n c-S)+(n-2) H \lambda_{1}+n H^{2} .
$$

If the condition in 1.3(b) holds on $M^{n}$, then the Ricci curvature of $M^{n}$ is positive and by Bonnet-Myers' theorem, $\pi_{1}(M)$ is finite. If $n=2$, we obtain $1.2(\mathrm{~b} 1)$ by the Gauss-Bonnet's formula. If $n=3$, since $\pi_{1}(\tilde{M})=\{0\}$, we have (b2) by Hamilton's theorem $[\mathrm{H}]$. Now let $n \geq 4$ and $M^{n}$ orientable when $n$ is even. Since

$$
\frac{n^{2} H^{2}}{n-1}+\frac{n(n-2)}{n-1} H \lambda_{1}+n c \leq \frac{n^{2} H^{2}}{n-2}+\frac{n(n-4)}{n-2} H \lambda_{1}+n c,
$$

it follows from part (a) that $H_{k}(M, \mathbf{Z})=\{0\}$ for $2 \leq k \leq n-2$. The above arguments applyed to the immersion $f \circ \pi: \tilde{M}^{n} \rightarrow Q_{c}^{n+m}$, where $\pi: \tilde{M}^{n} \rightarrow M^{n}$ is the covering map, tell us that $H_{k}(\tilde{M}, \mathbf{Z})=\{0\}$ for $2 \leq k \leq n-2$. Then (b2) is a consequence of Lemma 2.2.

Proof of Corollary 1.4. Under the hypothesis of 1.4, let $x \in M^{n}$ and let $v$ be a unit vector in $T_{x} M$. By (1) we have that

$$
\begin{aligned}
\operatorname{Ric}(v) & \geq \frac{n-1}{n}(n c-S)+n H^{2}+(n-2) H \lambda_{1} \\
& =\frac{n-1}{n}\left[-S+\left(n c+\frac{n^{2} H^{2}}{n-1}+\frac{n(n-2)}{n-1} H \lambda_{1}\right)\right] \\
& \geq \frac{n-1}{n}[-\sup (S)+\inf (T)]=-\left(\frac{n-1}{n}\right) \sup (S-T)=\delta>0 .
\end{aligned}
$$

Then $M^{n}$ is compact and the result follows from $1.3(\mathrm{~b})$. 


\section{Proofs of Theorem 1.5 and Corollary 1.6}

Proof of Theorem 1.5. Let $x \in M^{n}$ and let $v$ be a unit vector in $T_{x} M$. By using (1) and Lemma 2.1(b), it is easy to see that

$$
\frac{n}{n-1} \operatorname{Ric}(v) \geq A \geq B \geq C,
$$

where

$$
\begin{gathered}
A=\frac{n^{2} H^{2}}{n-1}+\frac{n(n-2)}{n-1} H \lambda_{1}+n-S, \\
B=n-S+2 n H^{2}-\frac{n(n-2) H}{n-1} \sqrt{\frac{n-1}{n}\left(S_{H}-n H^{2}\right)}, \\
C=n-S+2 n H^{2}-\frac{n(n-2) H}{n-1} \sqrt{\frac{n-1}{n}\left(S-n H^{2}\right),}
\end{gathered}
$$

where $S_{H}=\operatorname{tr} A_{\xi_{1}}^{2}$. We claim that $S \leq C(H)$ is equivalent to $C \geq 0$ and also $S=C(H)$ if and only if $C=0$. In fact, writing $S_{1}=S-n H^{2}$, we have

$$
\begin{aligned}
C & =n+n H^{2}-\left[\sqrt{S_{1}}+\frac{n(n-2)}{2(n-1)} \sqrt{\frac{n-1}{n}} H\right]^{2}+\frac{n(n-2)^{2} H^{2}}{4(n-1)} \\
& =(K-L)(K+L),
\end{aligned}
$$

where

$$
K=\sqrt{n+\frac{n^{3} H^{2}}{4(n-1)}}
$$

and

$$
L=\sqrt{S_{1}}+\frac{(n-2) H}{2} \sqrt{\frac{n}{n-1}} .
$$

Then $C \geq 0$ if and only if $K \geq L$, that is $S \leq C(H)$. By (21) we then have that the Ricci cuvature of $M^{n}$ is nonnegative.

(a) Let $\pi_{1}(M)$ be finite. Observe that $\tilde{M}^{n}$ is compact, in this case.

(a1) If $n=2$, it follows from the Gauss-Bonnet's formula that $M^{2}$ is diffeomorphic to $S^{2}$ or to $R P^{2}$.

(a2) If $n=3$, assume firstly that there exists a point $x$ in $M^{3}$ such that $\operatorname{Ric}(v)>0$ for all unit vector $v$ in $T_{x} M$. It follows then by Aubin's theorem that $M^{n}$ has a metric of positive Ricci curvature and, since $\pi_{1}(M)=\{0\}, M^{3}$ is diffeomorphic to $S^{3}$ by Hamilton's theorem. Suppose now that for each $x$ in $M^{n}$, there exists a unit vector $v \in T_{x} M$ such that $\operatorname{Ric}(v)=0$. Since $C \geq 0$, we have by (21) that $A=B=C=0$ and also, as it is easy to see, an equality occurs in (1) of Theorem 1.1 for each $x \in M$ and some unit vector $v \in T_{x} M$. Then $M^{3}$ 
is conformally flat and, since $\pi_{1}(M)=\{0\}$, we have by a theorem of Kuiper, see [D, p. 116], that $M^{3}$ is (conformally) diffeomorphic to $S^{3}$.

(a3) Let $n \geq 4$ and assume that $H \neq 0$ when $S=C(H)$. Since $A \geq B \geq$ $C \geq 0$, see (21), we have

$$
S \leq \frac{n^{2} H^{2}}{n-1}+\frac{n(n-2)}{n-1} H \lambda+n \leq \frac{n^{2} H^{2}}{n-2}+\frac{n(n-4)}{n-2} H \lambda_{1}+n
$$

on $M^{n}$. We claim that

$$
S<\frac{n^{2} H^{2}}{n-2}+\frac{n(n-4)}{n-2} H \lambda_{1}+n
$$

on $M^{n}$. In fact, if there exists a point $x$ in $M^{n}$ where (26) is an equality, then it follows from (25) that $H=0$ or $\lambda_{1}=H$ at $x$. It also follows from the equality in (26) that $A=B=C=0$ and so $S=C(H)$, that is, $H \neq 0$. Therefore $\lambda_{1}=H$ and by $A=0$, we obtain that $S=n+2 n H^{2}$ at $x$. On the other hand, by $S=n+2 n H^{2}$ and $C=0$, we obtain that $S=n H^{2}$ at $x$, a contradiction. This proves (26) and then, by Theorem $1.3(\mathrm{a})$ we have that $H_{i}(M, \mathbf{Z})=\{0\}$ for $i=2, \ldots, n-2$ and the same holds for $\tilde{M}^{n}$. Then (a3) follows from Lemma 2.2.

(b) If $\pi_{1}(M)$ is infinite then, for each $x$ in $M^{n}$ there exists a unit vector $v \in T_{x} M$ such that $\operatorname{Ric}(v)=0$. Otherwise by Aubin's theorem and BonnetMyers' theorem, we would have $\pi_{1}(M)$ finite. By (21) and $C \geq 0$ we have

$$
S=C(H), \quad A=B=C=0
$$

on $M^{n}$. Moreover, if $n \geq 3$ and $H \neq 0$, we have on $M^{n}$ that

$$
S=S_{H}, \quad \lambda_{1}=H-\sqrt{\frac{n-1}{n}\left(S_{H}-n H^{2}\right)} .
$$

(b1) If $n=2$, it is clear that $M^{2}$ is flat.

(b2) If $n \geq 3$, assume firstly that $H \neq 0$ on $M^{n}$. By (28) and Lemma 2.1(b), we can see that $A_{\xi_{1}}$ has two eigenvalues $\lambda_{1}$ and $\lambda_{2}$, where $\lambda_{1}$ has multiplicity at least $n-1$. Also $N_{1}=[\vec{H}]$ because $S=S_{H}$. Now we want to show that $\lambda_{1} \lambda_{2}=-1$. For this, observe that if $v$ is a unit vector in $T_{x} M$ and $\operatorname{Ric}(v)=0$, then the equality occurs in (1) for $x$ and $v$, and $A_{\xi_{1}} v=\lambda_{1} v$. Let $v_{1}=v, v_{2}, \ldots, v_{n}$ be an orthonormal basis of $T_{x} M$ such that $A_{\xi_{1}} v=\lambda_{1} v$ and $A_{\xi_{1}} v_{i}=\lambda_{2} v_{i}$ for $i \geq 2$. By the Gauss' equation we have

$$
\begin{aligned}
0 & =\operatorname{Ric}(v)=\sum_{i=2}^{n}\left(1+\left\langle\alpha\left(v_{1}, v_{1}\right), \alpha\left(v_{i}, v_{i}\right)\right\rangle-\left\|\alpha\left(v_{1}, v_{i}\right)\right\|^{2}\right) \\
& =(n-1)\left(\lambda_{1} \lambda_{2}+1\right),
\end{aligned}
$$

that is, $\lambda_{1} \lambda_{2} \equiv-1$. We claim that $N_{1}$ is parallel in the normal connection. Let $\xi_{1}, \ldots, \xi_{m}$ and $X_{1}, \ldots, X_{n}$ be local orthonormal frame fields, normal and tangent to $M^{n}$ respectively, chosen in a way that $\xi_{1}=\vec{H} / H$ and $A_{\xi_{1}} X_{1}=\lambda_{1} X_{1}, A_{\xi_{1}} X_{i}=$ 
$\lambda_{2} X_{i}, i \geq 2$. Since $\lambda_{1} \neq \lambda_{2}$, they are smooth functions on $M^{n}$. By the Codazzi equations

$$
\left(\nabla_{X_{1}}^{\perp} \alpha\right)\left(X_{i}, X_{1}\right)=\left(\nabla_{X_{i}}^{\perp} \alpha\right)\left(X_{1}, X_{1}\right), \quad\left(\nabla_{X_{1}}^{\perp} \alpha\right)\left(X_{i}, X_{i}\right)=\left(\nabla_{X_{i}}^{\perp} \alpha\right)\left(X_{1}, X_{i}\right),
$$

and by the fact that $N_{1}$ is spanned by $\vec{H}$, we obtain

$$
\begin{aligned}
& \lambda_{1} \nabla_{X_{i}}^{\perp} \xi_{1}=\left[\left(\lambda_{1}-\lambda_{2}\right)\left\langle\nabla_{X_{1}} X_{1}, X_{i}\right\rangle-X_{i}\left(\lambda_{1}\right)\right] \xi_{1}, \\
& \lambda_{2} \nabla_{X_{1}}^{\perp} \xi_{1}=\left[\left(\lambda_{2}-\lambda_{1}\right)\left\langle\nabla_{X_{1}} X_{i}, X_{1}\right\rangle-X_{1}\left(\lambda_{2}\right)\right] \xi_{1} .
\end{aligned}
$$

But the left sides of (29) cannot be parallel to $\xi_{1}$ unless $\nabla_{X_{i}}^{\perp} \xi_{1}=0$, for $i=$ $1,2, \ldots, n$. This proves our claim. By a well known result of Erbacher, the codimension $m$ of $f$ can be reduced to 1 and $f$ can be seen as an immersion of $M^{n}$ into $S^{n+1}$ where $A_{\xi_{1}}$ has two eigenvalues $\lambda_{1} \neq \lambda_{2}$. Now by a result of Ryan [R, p. 372], the distribution $T_{\lambda_{2}}:=\left\{X: A_{\xi_{1}} X=\lambda_{2} X\right\}$ is differentiable and involutive, and $X_{i}\left(\lambda_{2}\right)=0$ for $i \geq 2$. Since $\lambda_{1} \lambda_{2}=-1$, we also have that $X_{i}\left(\lambda_{1}\right)=0$ for $i \geq 2$. Taking this to (29), we see that $\left\langle\nabla_{X_{1}}^{X_{1}}, X_{i}\right\rangle=0$ for $i \geq 2$ and, since $\left\langle\nabla_{X_{1}}^{X_{1}}, X_{i}\right\rangle=0$, this shows that the orthogonal distribution $T_{\lambda_{2}}^{\perp}=$ $\left\{\xi_{1}: A_{\xi_{1}} X=\lambda_{1} X\right\}$ is totally geodesic. We then have a compact manifold $M^{n}$ with nonnegative Ricci curvature and with a codimension one foliation, defined by $T_{\lambda_{2}}$, whose orthogonal distribution $T_{\lambda_{2}}^{\perp}$ is totally geodesic. It follows immediately from Corollary 2 of $[\mathrm{BW}]$ that $T_{\lambda_{2}}$ is also totally geodesic and then $\left\langle\nabla_{X_{i}} X_{i}, X_{1}\right\rangle=0$ for $i \geq 2$. Again by (29) we conclude that $X_{1}\left(\lambda_{2}\right)=0$. This proves that $\lambda_{1}$ and $\lambda_{2}$ are constant on $M^{n}$. Clearly $f$ has constant mean curvature $H \neq 0$ and $S=C(H)$. Now (b2) follows, in this case, from the theorem in $[\mathrm{AC}]$ or $[\mathrm{Xu}]$ quoted in the Introduction.

Suppose now that there exists a point $x_{0}$ in $M^{n}$ such that $H\left(x_{0}\right)=0$. Since $A=B=C=0$ on $M^{n},(1)$ is an equality everywhere. By Theorem $1.1, R^{\perp}=0$ and $M^{n}$ is conformally flat. On the other hand, $S\left(x_{0}\right)=n$ because $H\left(x_{0}\right)=0$ and then $\tau\left(x_{0}\right)=n(n-2)$ by $(10)$, that is, $M^{n}$ cannot be flat. Since $\pi_{1}(M)$ is infinite, we can now use the same arguments of the proof of Theorem 1 of $[\mathrm{N}$, p. 259], to conclude that $\tilde{M}^{n}=\boldsymbol{R}^{n} \times S_{c_{2}}^{n-1}$, for some $c_{2}>0$. This shows that $\tilde{M}^{n}$ has constant scalar curvature and so $M^{n}$ also, that is, $\tau \equiv n(n-2)$. Again by (10), $S=n^{2} H^{2}+n$ on $M^{n}$ and combining this with $S=C(H)$, we obtain that $H \equiv 0, S \equiv n$. The result (b2) now follows from Corollary 1.2, in this case.

Proof of Corollary 1.6. Let $S \leq 2 \sqrt{(n-1)}$ on $M^{n}$. By (24), we have

$$
C=D \geq E \geq 0
$$

on $M^{n}$, where

$$
\begin{aligned}
D & =n-\frac{n S}{2 \sqrt{n-1}}+\frac{a^{2}}{2 \sqrt{n-1}}, \\
a & =(\sqrt{n-1}+1) \sqrt{n} H-(\sqrt{n-1}-1) \sqrt{S-n H^{2}},
\end{aligned}
$$

and 


$$
E=n\left(1-\frac{S}{2 \sqrt{n-1}}\right)
$$

Since $C \geq 0$, we have that $M^{n}$ has nonnegative Ricci curvature and $S \leq C(H)$ on $M^{n}$. We have two possibilities, (a) and (b):

(a) There exists a point $x$ in $M^{n}$ such that $\operatorname{Ric}(v)>0$ for all unit $v$ in $T_{x} M$. In this case, it follows from Aubin's theorem and Bonnet-Myers' theorem that $M^{n}$ has a metric of positive Ricci curvature, $M^{n}$ is compact and $\pi_{1}(M)$ is finite. If $n=2$ or $n=3$, then (a1) and (a2) follows from the same arguments as in $1.5(\mathrm{a} 1)(\mathrm{a} 2)$. For (a3), let $n \geq 4$ and observe that $H \neq 0$ when $S=C(H)$. In fact, if there exists $x_{0}$ in $M^{n}$ with $S=C(H)$ and $H=0$, then $C\left(x_{0}\right)=0$ and also $D\left(x_{0}\right)=E\left(x_{0}\right)=0$, by $(30)$. This shows that $0=S=2 \sqrt{n-1}$ at $x_{0}$, which is a contradiction. Then (a3) follows from 1.5(a3).

(b) For the case (b), using (30) we obtain $A=B=C=D=E=0$ on $M^{n}$. Then $S \equiv 2 \sqrt{n-1}$ and $n^{2} H^{2} \equiv n \sqrt{n-1}-2(n-1)$. (b1) If $n=2$, then $M^{2}$ is flat and $H=0$. In particular, if $m=1$, it follows from [CCK] or [L] that $M^{2}$ is isometric to $S_{2}^{1} \times S_{2}^{1}$. (b2) Let $n \geq 3$. In this case, it follows from $B=0$ that $S=S_{H}$ on $M^{n}$ and therefore $N_{1}$ is spanned by $\vec{H}$. We now imitate the proof of $1.5(\mathrm{~b})$ to show that $f$ can be seen as an isometric immersion of $M^{n}$ into $S^{n+1}$ with constant $H \neq 0$ and $S=2 \sqrt{n-1}$ on $M^{n}$; (b2) is now a direct consequence of the result in $[\mathrm{Ho}]$ mentioned in the Introduction.

Acknowledgements. This work is part of the doctoral thesis of the second author presented at IME-USP under the advising of the first author. E. A. Costa thanks the Brazilian agency CAPES for the finantial support. Both authors thank to F. Brito, F. Mercuri and P. Simões for their comments and suggestions on this work, and the referee for valuable suggestions that improved the paper.

\section{REFERENCES}

[A] T. Aubin, Metriques Riemanniennes et courbure, J. Differential Geom., 4 (1970), 385-424.

[AC] H. Alencar AND M. Do CARmo, Hypersurfaces with constant mean curvature in spheres, Proc. Amer. Math. Soc., 120 (1994), 1223-1228.

[B] G. E. Bredon, Topology and Geometry, Springer-Verlag, New York, 1993.

[BW] F. G. B. BRito AND P. L. WALCZAK, Totally geodesic foliations with integrable normal bundles, Bol. Soc. Brasil. Mat., 17 (1986), 41-46.

[CCK] S. S. Chern, M. do Carmo and S. Kobayashi, Minimal submanifolds of the sphere with second fundamental form of constant lenght, Functional Analysis and Related Fields (F. E. Browder ed.), Springer-Verlag, New York, 1970, 59-75.

[CD] M. DO CARMo AND M. DAJCZER, Rotation hypersurfaces in spaces of constant cuvature, Trans. Amer. Math. Soc., 277 (1983), 685-709.

[CG] J. Cheeger and D. Gromoll, The splitting theorem for manifolds of non-negative Ricci curvature, J. Differential Geom., 6 (1971), 119-128.

[CN] Q. M. Cheng and H. Nakagawa, Totally umbilic hypersurfaces, Hiroshima Math. J., 20 (1990), 1-10. 
[CY] B. Y. Chen AND K. Yano, Sous-varietés localement conformes a un espace euclidean, C.R. Acad. Sci. Paris Sér. A-B, 275 (1972), 123-126.

[D] M. Dajczer, Submanifolds and Isometric Immersions, Publish or Perish, Inc., Houston, 1990.

[H] R. S. Hamilton, Three manifolds with positive Ricci curvature, J. Differential Geom., 17 (1982), 255-306.

[Ho] Z.-H. Hou, A pinching problem on submanifolds with parallel mean curvature vector, Kodai Math. J., 21 (1998), 35-45.

[K] K. Kenmotsu, Some remarks on minimal submanifolds, Tôhoku Math. J., 22 (1970), 240 248.

[L] H. B. Lawson, Local rigidity theorems for minimal hypersurfaces, Ann. of Math., 89 (1969), 187-197.

[Le1] P. F. Leung, Minimal submanifolds in a sphere, Math. Z., 183 (1983), 75-86.

[Le2] P. F. Leung, On a relation between the topology and the intrinsic and extrinsic geometries of a compact submanifold, Proc. Edinburgh Math. Soc., 28 (1985), 305-311.

[Le3] P. F. Leung, An estimate on the Ricci curvature of a submanifold and some applications, Proc. Amer. Math. Soc., 114 (1992), 1051-1061.

[LS] H. B. LAWSON AND J. Simons, On stable currents and their applications to global problems in real and complex geometry, Ann. of Math., 98 (1973), 427-450.

[N] M. H. Noronha, Some compact conformally flat manifolds with nonnegative scalar curvature, Geom. Dedicata, 47 (1993), 225-268.

[R] P. Ryan, Homogeneity and some curvature conditions for hypersurfaces, Tôhoku Math. J., 21 (1969), 363-388.

[S] J. Simons, Minimal varieties in riemannian manifolds, Ann. of Math., 88 (1968), 62-105.

[Sj] D. SJERve, Homology spheres which are covered by spheres, J. London Math. Soc. (2), 6 (1973), 333-336.

[Sp] E. H. Spanier, Algebraic Topology, McGraw-Hill, New York, 1966.

[SX] K. Shiohama And H. Xu, The topological sphere theorem for complete submanifolds, Compositio Math., 107 (1997), 221-232.

[X] Y. L. XIN, An application of integral currents to the vanishing theorems, Sci. Sinica Ser. A, 27 (1984), 233-241.

$[\mathrm{Xu}] \mathrm{H} . \mathrm{Xu}, \mathrm{A}$ rigidity theorem for submanifolds with parallel mean curvature vector in a sphere, Arch. Math., 61 (1993), 489-496.

[W] S. W. WeI, Generalized idea of Synge and its applications to topology and calculus of variations in positively curved manifolds, Nonlinear Functional Analysis and Its Applications (F. E. Browder ed.), Proc. Sympos. Pure Math. 45-2, AMS, Providence, 1986, 533539.

\author{
InSTituto de Matemática e Estatística \\ Universidade de São Paulo \\ Rua do Matão 1010, Cidade UniversitáRIA \\ 05508-900-SÃo PaUlo-SP-BraZIL \\ e-mail: asperti@ime.usp.br \\ Instituto De Matemática \\ UNIVERSIDADE FEDERAL DA BAHIA \\ Rua Ademar de Barros s/N-Campus de Ondina \\ 40170-110-SALVADOR-BA-BRAZIL \\ e-mail: ezio@ufba.br
}

